\title{
Vescalagin and Castalagin Present Bactericidal Activity toward Methicillin-Resistant Bacteria
}

\author{
Ana R. Araújo,* Ana C. Araújo, Rui L. Reis, and Ricardo A. Pires* \\ Cite This: https://dx.doi.org/10.1021/acsbiomaterials.0c01698 \\ Read Online
}

ABSTRACT: Polyphenols have been extensively exploited in the biomedical field because of their wide range of bioactive properties and historical use as traditional medicines. They typically present antioxidant, antimicrobial, antiamyloidogenic, and/or antitumor activities. In particular, cork water extracts and their components, have been previously reported to present antioxidant and antiamyloidogenic properties. On the basis of this knowledge, we tested cork water extract (CWE), cork water enriched extract (CWE-E), vescalagin/castalagin (two of the main polyphenols present in CWE and CWE-E) for their antibacterial activity against four bacterial strains, namely, methicillinresistant Staphylococcus epidermidis (MRSE), Staphylococcus aureus (SA), methicillin-resistant Staphylococcus aureus (MRSA), and Pseudomonas aeruginosa (PA). Vescalagin and castalagin presented bactericidal activity against all the tested bacterial strains, in particular toward the methicillin-resistant ones, i.e., MRSA and MRSE, as well as the ability to inhibit the formation of biofilms and to disrupt preformed ones. Moreover, vescalagin/castalagin seem to modulate the normal assembly of the peptidoglycans at the bacteria surface, promoting the disruption of their cell wall, leading to bacterial cell death. We also demonstrate that vescalagin/castalagin can be loaded into alginate hydrogels to generate antibacterial biomaterials that are not toxic to eukaryotic cells.

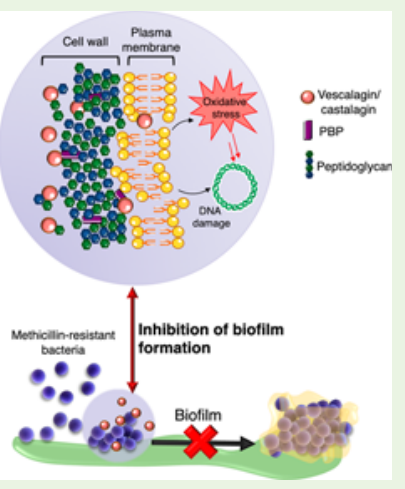

KEYWORDS: cork, vescalagin, castalagin, methicillin-resistant Staphylococcus epidermidis, Staphylococcus aureus, methicillin-resistant Staphylococcus aureus, Pseudomonas aeruginosa

\section{INTRODUCTION}

Pathogenic bacteria are a serious health concern in modern society, as they present increasing resistance to the most common antibiotics. ${ }^{1}$ They are associated with chronic infections and severe inflammation due to their ability to create a physical and chemical barrier (through the extracellular matrix) to external factors, enhancing their resistance to therapeutic strategies. ${ }^{2}$ They grow under both static and continuous flow conditions in a dense layer of cells within an elaborate matrix that harbors various types of biopolymers including phenol-soluble modulins (PSMs), alginate, exopolysaccharides, extracellular DNA, and different proteins able to generate amyloid-like fibers (i.e., curli or fimbriae), which in turn are used by bacteria to aggregate, form microcolonies, and generate biofilms. ${ }^{3-5}$

Biofilms are extremely common, being present in water pipes (inner surfaces), on dental plaque, in lung infections, and general infections related to the use of medical devices. ${ }^{6}$ They are described as compact aggregates of bacteria attached to a surface and surrounded by a mesh of extracellular polymeric substances (EPS) that are produced over time (composed by approximately $5-25 \%$ of bacterial cells and $75-95 \%$ of glycocalyx matrix). ${ }^{7}$ The bacterial surface is also composed by amyloid-like fibers that play a role in adherence, as well as colanic acid, an exopolysaccharide involved in aggregation. ${ }^{8}$ Biofilms enhance bacterial survival, as they are protected from environmental stress such as nutrient deprivation, unphysiological temperatures, and $\mathrm{pH}$ changes, making them more resistant to detachment, oxygen radicals, disinfectants, and antibiotics. Consequently, bacteria embedded in mature biofilms can tolerate antimicrobial agents at concentrations 10-1000x higher than the ones needed to kill genetically equivalent individual bacteria. ${ }^{9}$ Biofilms are not required for bacteria survival; it is an adaptation that enhances survival, metabolism, and propagation of bacteria, especially under adverse conditions. In contrast, circulating bacteria are able to colonize new microenvironments, however they present a lower chance of survival. ${ }^{8}$ Therefore, strategies that block the production of adherent proteins (e.g., able to form curli and amyloid-like fibers) or to inhibit their activity, are extremely relevant to reduce the formation of biofilms from different types of pathogenic bacteria that are resistant to antibiotics, e.g., methicillin-resistant ones.

Importantly, bacteria are divided into Gram-positive and Gram-negative, a classification that is related to the chemical/

Received: December 6, 2020

Accepted: January 29, 2021 
physical composition of the cell membrane that protect their cytoplasm from external aggression. ${ }^{10}$ Although Gram-positive bacteria have a thick layer of peptidoglycans, ${ }^{11}$ in the Gramnegative bacteria this layer is thinner; however, they present an additional outer membrane that contains a unique type of component, i.e., lipopolysaccharides, and an inner membrane composed of proteins and phospholipids. ${ }^{3,12,13}$

Polyphenols have been studied as alternative antibacterial agents. ${ }^{14}$ They are essential components of plants where they can be found in a series of different roles. ${ }^{15}$ They are not involved in the normal growth and maturation of plants (as they are secondary metabolites), however, they have a critical role in the plants' defense mechanisms against viruses, bacteria, or fungi. ${ }^{16}$ The antioxidant and antimicrobial activities of these molecules are related to the number of phenyl rings and the number of conjugated hydroxyl groups (e.g., galloyl units). ${ }^{17}$ Polyphenols (e.g., epigallocatechin gallate (EGCG), resveratrol, etc.) or tannins have been tested for a range of different pathologies (i.e., inflammation, cardiovascular diseases, cancer, and neurodegenerative disorders, among others), or as molecules that are able to enhance the human immune response mechanisms. ${ }^{18-20}$

Cork is a relevant natural source of polyphenols. In fact, we previously demonstrated that it is possible to extract a series of phenolic compounds using hot water, i.e., cork water extract (CWE), with important antioxidant activity. ${ }^{21}$ On the basis of these results, we also showed that it is possible to use CWE or vescalagin/castalagin (isolated from CWE) to protect DNA from UV-mediated damage and subsequent cell death, ${ }^{22}$ or as antiamyloidogenic agents. ${ }^{23}$

In the context of antibacterial activity, the mechanism of action of most polyphenols is reported to be related to their ability to target the bacterial membrane, interacting with the lipids and its subsequent permeabilization. ${ }^{24}$ Of note, in a previous study, we demonstrated that vescalagin/castalagin was able to interact with the amyloidogenic peptide $\mathrm{A} \beta 42$, inhibiting the generation of cytotoxic supramolecular forms and modulating its activity. Importantly, amyloids are highly abundant in the matrices of biofilms of diverse bacterial species, sharing a common structural feature, i.e., cross- $\beta$ sheets. ${ }^{25}$ These bacterial amyloid-like fibers mediate adhesion and promote biofilm formation, as they are a relevant target to inhibit the formation of biofilms. ${ }^{26}$

Herein, we used the knowledge from our previous results and explored the antibacterial capacity of cork-based polyphenols, namely, the CWE, vescalagin, and castalagin (Figure 1). We also tested the enrichment of CWE in vescalagin, through the use of an additional subsequent extraction with ethanol (CWE-E). We tested these extracts/ polyphenols against four different pathogenic bacteria strains, methicillin-resistant Staphylococcus epidermidis (MRSE, ATCC 35984), Staphylococcus aureus (SA, ATCC 25923), methicillinresistant Staphylococcus aureus (MRSA, ATCC 700698), and Pseudomonas aeruginosa (PA, ATCC 27853).

\section{RESULTS AND DISCUSSION}

We started by extracting CWE and CWE-E, as well as isolating the polyphenols vescalagin/castalagin (characterization is presented in Figures $\mathrm{S} 1-\mathrm{S} 5)$. We then evaluated their antioxidant activity using the DPPH assay (Figure S6) to understand if there was a relationship between antioxidant and antibacterial activities. The second ethanol extraction, yielding CWE-E from the CWE extract, allowed an enrichment in the
A
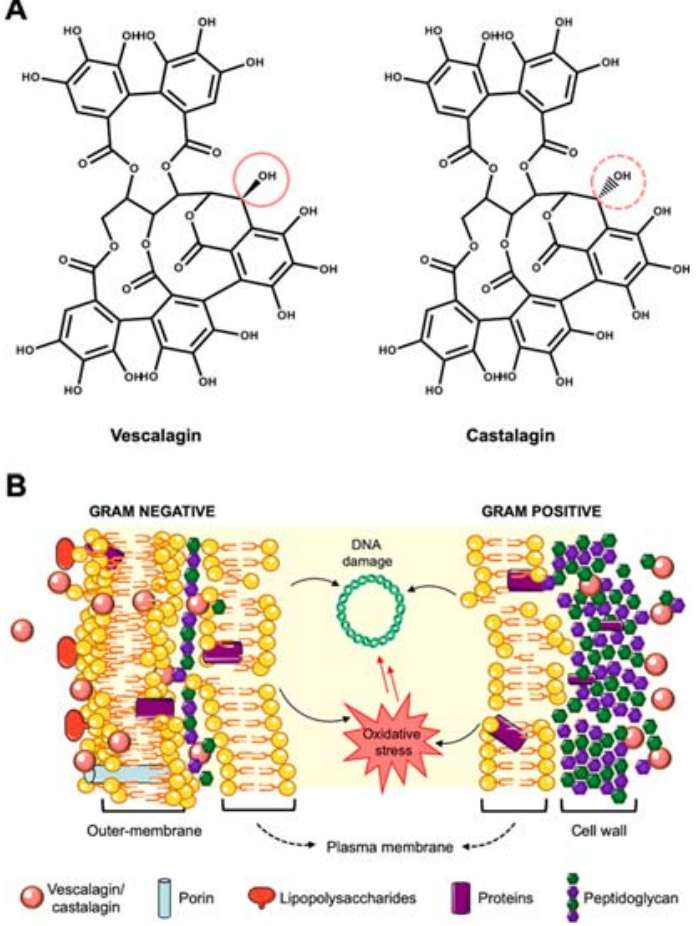

Figure 1. (A) Chemical structure of the polyphenols vescalagin and castalagin purified from CWE. (B) Schematic presentation of the mechanism of action of vescalagin/castalagin: (i) Gram-negative, antibacterial activity through the diffusion into a region near the peptidoglycans layer; (ii) Gram-positive,direct binding of vescalagin/ castalagin to the peptidoglycan of the bacterial cell wall and its subsequent disruption.

vescalagin content, and this is reflected in the antioxidant potential of the CWE-E $(\sim 10 \%$ higher than the original CWE). Of note, the purified vescalagin presented $\sim 30 \%$ higher antioxidant activity than CWE, whereas in the case of castalagin, an $\sim 37 \%$ increment was observed. These results could be directly related to antibacterial activity: (i) due to the anti- and pro-oxidant capacity of the galloyl moieties present in the molecular structure of the polyphenols (these molecules can oxidize readily in aqueous solution, acting as pro-oxidants, i.e., producing reactive oxygen species and a complex mixture of quinones, which are potentially cytotoxic), ${ }^{27}$ thus, leading to the formation of peroxides and their restructuring to form $o$ diphenols (increasing the free-radical scavenging capacity); ${ }^{28}$ (ii) the formation of $o$-diphenols is linked with the polyphenols' ability to complex with proteins (at the surface of the cell wall); ${ }^{29}$ (iii) polyphenols are reported to interact with the polar regions of the lipid bilayer, promoting lipid peroxidation and therefore inhibiting bacterial growth; ${ }^{16}$ and (iv) it has been reported that their antioxidant activity is linked to the ability of these molecules to promote metal-ion deficiency based on their chelating ability, leading to the inhibition of bacterial enzymatic activity due to substrate deprivation. $^{30}$

To evaluate if the cork-derived extracts/polyphenols are able to act as antibacterial agents, we started with disc diffusion tests (Figures S7 and S8 and Table S1). The results showed that vescalagin/castalagin presented clear antibacterial activity toward all the Gram-positive bacteria. We then evaluated their minimum inhibitory concentration (MIC, Table 1) and minimum bactericidal concentration (MBC, Table S2) 
Table 1. Minimum Inhibitory Concentration (MIC, mg/ $\mathrm{mL}$ ) of Each Extract/Polyphenol, i.e., CWE, CWE-E, Vescalagin, and Castalagin, toward the Four Tested Strains of Bacteria, Methicillin-ResistantStaphylococcus epidermidis (MRSE), Staphylococcus aureus (SA), MethicillinResistantStaphylococcus aureus (MRSA), and Pseudomonas aeruginosa (PA)

\begin{tabular}{clccc} 
& \multicolumn{4}{c}{ Extract/polyphenol MIC $(\mathrm{mg} / \mathrm{mL})$} \\
\cline { 2 - 5 } Strain of bacteria & CWE & CWE-E & vescalagin & castalagin \\
\hline MRSE & 0.500 & 0.500 & 0.125 & 0.250 \\
SA & 2.000 & 1.000 & 0.500 & 0.500 \\
MRSA & 2.000 & 1.000 & 0.125 & 0.125 \\
PA & $\mathrm{NI}^{a}$ & $\mathrm{NI}^{a}$ & 1.000 & 1.000
\end{tabular}

${ }^{a} \mathrm{NI}$ : No inhibition at concentrations up to $2.50 \mathrm{mg} / \mathrm{mL}$.

towards the same bacterial strains, i.e., MRSE, SA, and MRSA. In general, all the tested bacteria are affected by the presence of the cork extracts/polyphenols. Importantly, vescalagin/castalagin seems to be the components present in the CWE/CWE$\mathrm{E}$ that present higher antibacterial activity, with MIC values between 0.125 and $0.250 \mathrm{mg} / \mathrm{mL}$ and MBCs between 0.250 and $1.000 \mathrm{mg} / \mathrm{mL}$. Of note, it was observed a higher sensitivity of the methicillin-resistant strains to vescalagin/castalagin than the nonresistant strain.

In the case of the Gram-negative bacterium PA, it presented a higher MIC/MBC (only measured for the purified compounds, i.e., vescalagin/castalagin, Table 1 and Table S2). It seems that the outer membrane of the Gram-negative bacteria renders additional resistance to the tested compounds. In contrast, our results are consistent with the high efficiency of vescalagin/castalagin to disrupt the peptidoglycan layer of the cell wall from the Gram-positive bacteria. In addition, overall, for both Gram-positive and Gram-negative bacteria, we observed a direct relation between antioxidant and antibacterial activity, as CWE-E presented lower MBCs than CWE, which correlates with its significantly higher antioxidant activity, suggesting that it is contributing to its capacity to promote bacterial cell death.

We then used the Live/Dead assay (Figures 2 and 3 and Figure S9) to confirm the MIC/MBC results. In this case, it is clear that vescalagin and castalagin effectively suppress the bacteria proliferation at the MIC concentrations (presented in Table 1), drastically reducing the percentage of live bacteria cells (Figure 3). Overall, and in accordance with the disc diffusion, MIC, and MBC results, it is surprising that vescalagin and castalagin present lower MICs and MBCs against MRSE and MRSA than against SA. Hence, we hypothesize that the mechanism of action of vescalagin/castalagin is partially linked to their capacity to interact with the proteins at the surface of the bacteria cell wall responsible for the methicillin resistance.

If we compare the MRSA and SA bacterial strains, their main difference is based on the capacity of MRSA (and not SA) to produce an additional penicillin-binding protein, PBP2a (as a result of an extensive use of $\beta$-lactam class of antibiotics, these strains became resistant to an entire class of $\beta$-lactam-based antibiotics). Although PBPs make MRSA and MRSE resistant to common antibiotics, they are also responsible for the final step of the synthesis of peptidoglycans (covalently cross-linked units of $\mathrm{N}$-acetylglucosamine and $\mathrm{N}$-acetylmuramic acid). ${ }^{4}$ Specifically, the serine nucleophile of the PBP2a active site changes its supramolecular assembly to diminish the reactivity against $\beta$ - lactam.

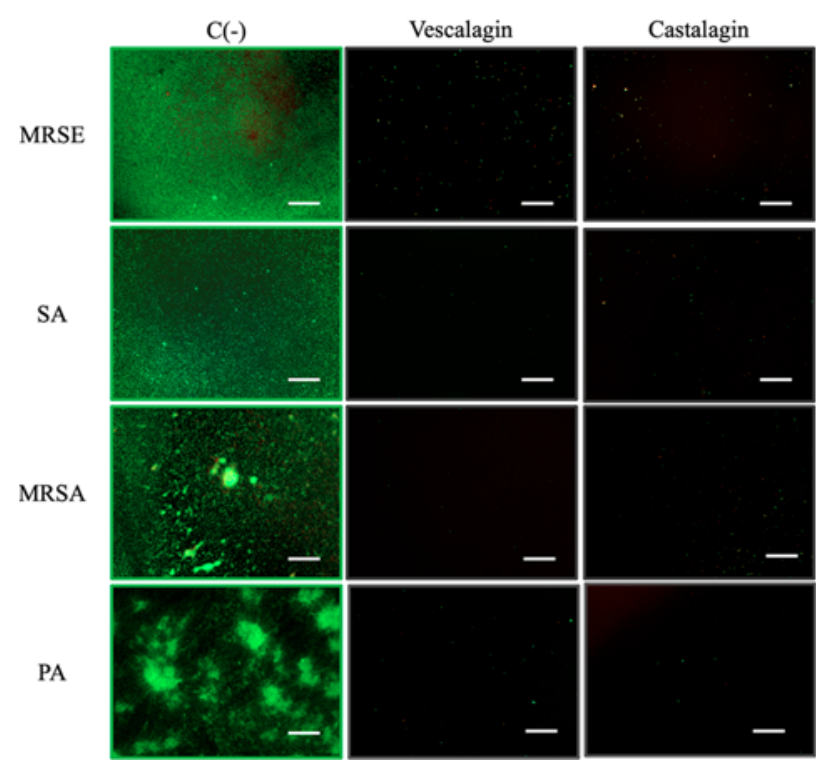

Figure 2. Representative Live/Dead images (in green live cells and in red dead cells) showing the antibacterial activity of vescalagin and castalagin (at their MIC concentration) toward methicillin-resistant Staphylococcus epidermidis (MRSE), Staphylococcus aureus (SA), methicillin-resistant Staphylococcus aureus (MRSA), and Pseudomonas aeruginosa $(\mathrm{PA})$; scale bar $=200 \mu \mathrm{m}$.
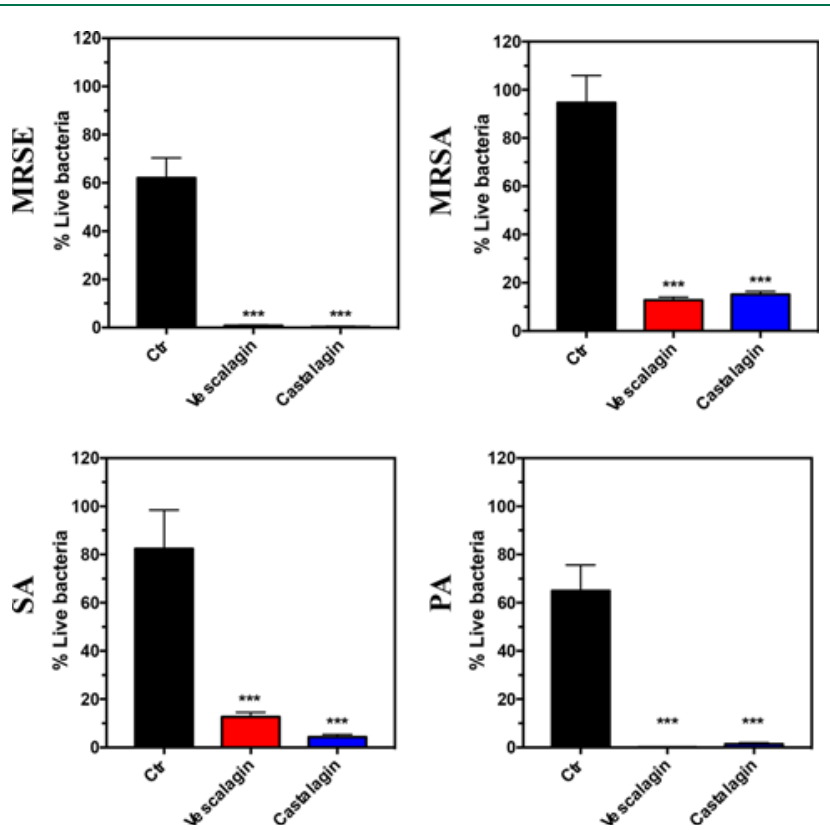

Figure 3. Quantification of live bacteria (as a percentage of the total live + dead ones found in the control sample). Quantification was executed using the fluorescence images obtained from the samples stained with BacLight Viability Kit (at the respective MIC concentration). Statistical significance: $* * * p<0.001, * * p<0.01$, and $* p<0.05$ (vs Ctr, i.e., bacterial culture in the absence of vescalagin/castalagin); $n=3$.

Also, the loop in the PBP2a protein protects its active site from the $\beta$-lactam antibiotics. ${ }^{31}$ In addition, some methicillinresistant cell-wall-anchored proteins are composed by several connecting subdomains folded into $\beta$-sheet rich regions. ${ }^{32}$ These supramolecular arrangements can explain the lower $\mathrm{MIC}$ values for vescalagin toward methicillin-resistant bacteria 
as being due to its proven higher antiamyloidogenic capacity when compared with castalagin. ${ }^{23,33}$

In the case of PA, the efficacy of CWE, CWE-E, and vescalagin/castalagin is lower. It is important to consider that PA is the only Gram-negative bacterium that we tested. This lower activity of the cork-derived extracts/polyphenols is, as previously suggested, probably due the different organization of its outer membrane. However, it is also important to consider that PA is associated with serious illnesses (e.g., infections such as ventilator-associated pneumonia) and presents complex antibiotic resistance mechanisms, ${ }^{34}$ which might be also at play in its resistance toward vescalagin/ castalagin.

The formation of biofilms increases the capacity of bacteria to resist external aggressions. To evaluate if the extracts/ polyphenols were able to act under these circumstances and if they were able to target proteins that mediate adhesion and biofilm formation, we used scanning electron microscopy (SEM, Figure 4) to visualize the bacteria in the presence and

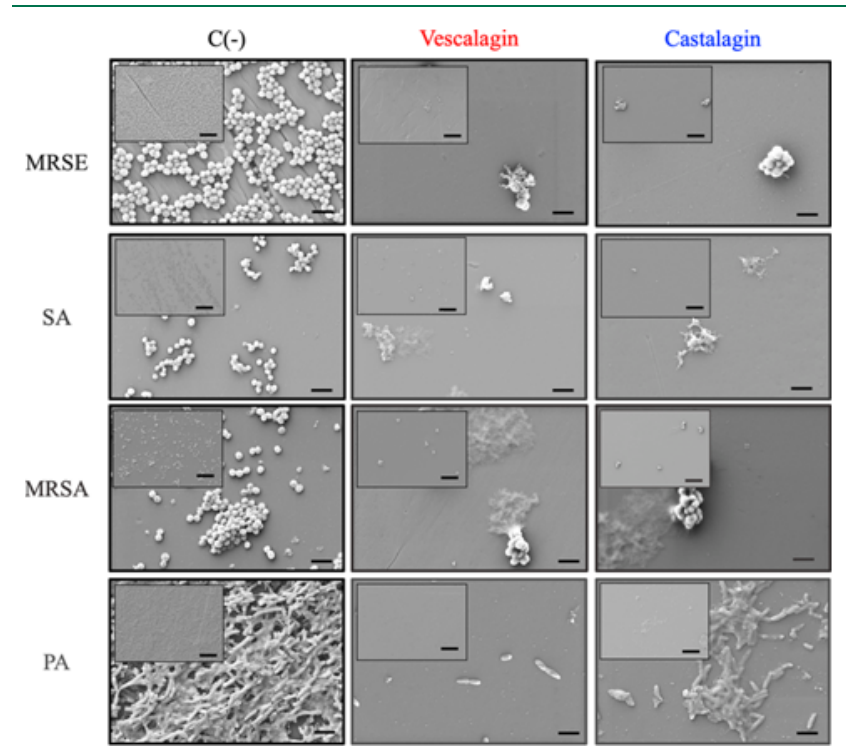

Figure 4. Representative SEM images showing a deterioration of cell wall of each strain of bacteria, namely, methicillin-resistant Staphylococcus epidermidis (MRSE); Staphylococcus aureus (SA), methicillinresistant Staphylococcus aureus (MRSA), and Pseudomonas aeruginosa (PA). Scale bar $=5 \mu \mathrm{m}$ (insets $=20 \mu \mathrm{m}$ ); experiments executed at the corresponding MIC.

absence of the extracts/polyphenols. Both vescalagin and castalagin clearly reduced the number of bacteria and altered their morphology by promoting the disruption of the bacterial cell wall. In addition, their extracellular matrix also appears unstructured, confirming the MIC/MBC data. After showing that vescalagin/castalagin reduce the formation of biofilms, we tested if they were also able to act once the biofilms are already formed (Figure 5). In this case, we observed a clear reduction of the preformed biofilms generated by all the tested strains of bacteria, using the MICs of vescalagin/castalagin. Importantly, in the cases of the Gram-positive bacteria, i.e., MRSE, MRSA, and SA, we were able to observe this reduction even at concentrations below their MICs.

To evaluate if vescalagin/castalagin could interfere with the formation of $\beta$-sheet rich structures essential for cell survival, we used the Congo red agar (CRA) assay, ${ }^{35}$ as it is usually sensitive to the presence of amyloid-like protein structures.
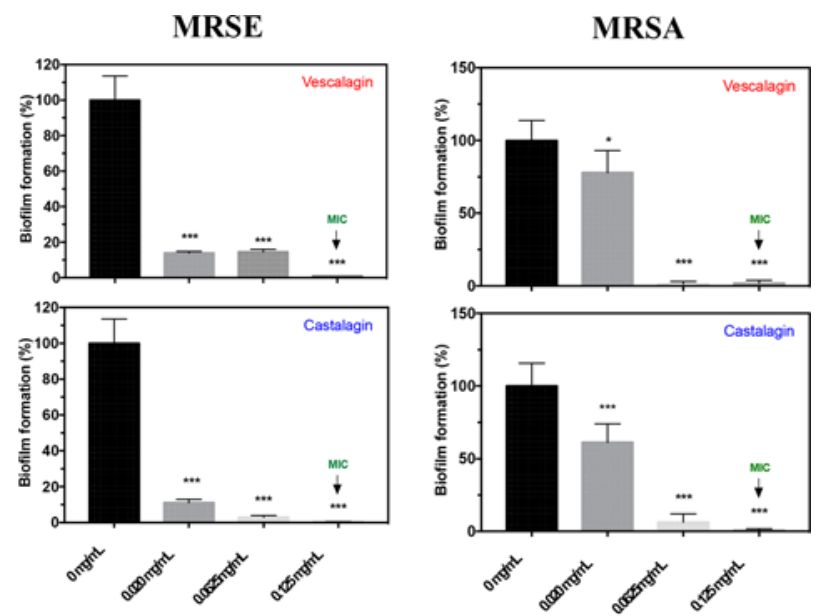

SA

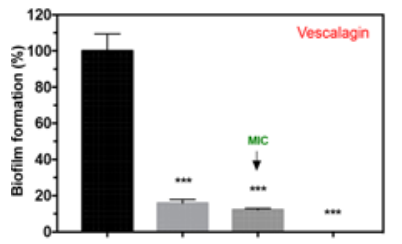

PA
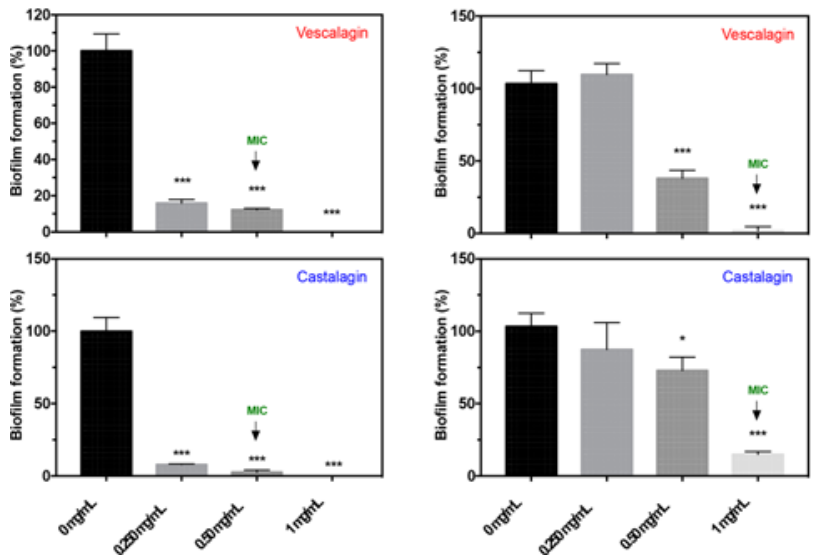

Figure 5. Capacity of vescalagin and castalagin (at different concentrations) to disrupt preformed biofilms from methicillinresistant, Staphylococcus epidermidis (MRSE), Staphylococcus aureus (SA), methicillin-resistant Staphylococcus aureus (MRSA), and Pseudomonas aeruginosa (PA). The relative formation of biofilm was quantified by safranin staining. Error bars $=\mathrm{SD}, * p<0.05$ and *** $p<0.001$ vs " $0 \mathrm{mg} / \mathrm{mL}$ ”; $n=4$.

Congo red is able to bind to these types of supramolecular structures, namely, the ones generated by polysaccharide intercellular adhesin, i.e., PIA or PSMs (in Staphylococcus strains), and exopolysaccharides, such as Pel and Psl in $\mathrm{PA}^{36-40}$ In general, the formation of light-colored colonies of MRSE, MRSA, and SA is associated with the inexistence of biofilm (and reduced concentration of $\beta$-sheet structures), whereas darker ones are consistent with formation of biofilm (rich in $\beta$-sheet structures). ${ }^{41}$

In the CRA assay, we used a concentration below the MIC values (presented in Table 1) because at those concentrations (and above) it was observed a strong (and dose-dependent) inhibition of the colony formation, being difficult to extract relevant conclusions (Figures S11-S14). The CRA assay (Figure 6) showed that both vescalagin and castalagin caused a strong inhibition of the formation of biofilm (and cross $\beta$-sheet structures) by the MRSE, SA and MRSA strains. In the case of $\mathrm{PA}$, its biofilm is stained light red by Congo red (Figure 6D). Reports have shown that, in this case, it is difficult the generate biofilms during CRA, and this test might not be suitable for identifying the exopolysaccharide layer produced by nonfermenting Gram-negative bacteria, such as PA. ${ }^{42,43}$ We obtained a light pink color mesh, probably due to the 

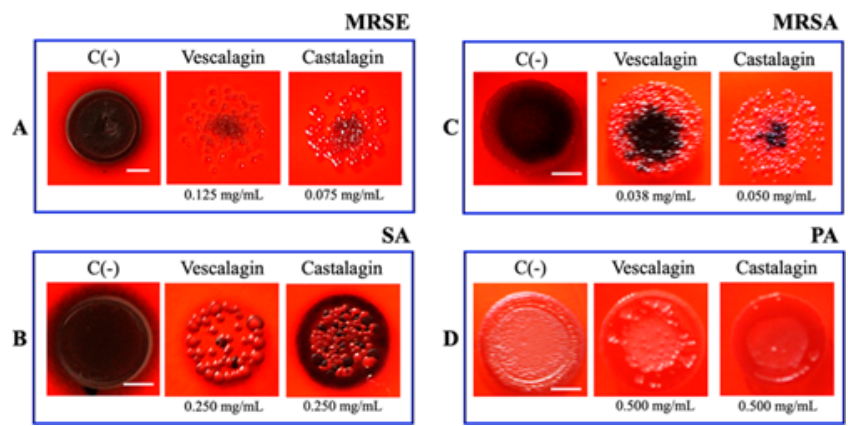

Figure 6. Representative Congo red assay images showing a decrease in the dark pigmentation consistent with a decrease of amyloid-like protein presentations for the tested strains of bacteria, namely, methicillin-resistant Staphylococcus epidermidis (MRSE), Staphylococcus aureus (SA), methicillin-resistant Staphylococcus aureus (MRSA), and Pseudomonas aeruginosa (PA). Scale bar $=5 \mathrm{~mm}$.

interference in the cell-to-cell communication process (i.e., quorum sensing). Under these conditions, it has been reported that PA cannot catabolize glucose, and is thus unable to synthesize the biofilm components. ${ }^{44,45}$ Our results are also consistent with the ability of vescalagin/castalagin to efficiently remodel the adherence proteins (i.e., PIA and PSMs), leading to bacterial cell death and reduction of the biofilm formation by PA.

Interestingly, vescalagin/castalagin are more efficient at inhibiting the growth and killing methicillin-resistant strains. In fact, Stapleton et al. ${ }^{46}$ showed that, at low concentrations (i.e., $0.025 \mathrm{mg} / \mathrm{mL}$ ), EGCG can reduce the amount of MRSA by its direct binding to the peptidoglycan layer at the $\mathrm{N}$ acetylglucosamine and $\mathrm{N}$-acetylmuramic acid residues, but only if it acts synergistically with the $\beta$-lactam oxacillin (antibiotic of the penicillin class). ${ }^{46}$ This combinatorial strategy has been successfully tested not only with EGCG but also with other polyphenols, e.g. (+)-catechin and (-)-epicatechin gallate, confirming their ability to enhance the activity of $\beta$-lactam antibiotics. In addition, the MIC concentration obtained for vescalagin is $4 \times$ lower than the one found for EGCG against the other methicillin-resistant bacterium, i.e., MRSE. ${ }^{47}$ As mention before, these strains express the PBP2a protein that acts as cross-linker of the peptidoglycan layer. Therefore, it is likely that vescalagin/ castalagin is a strong PBP2a inhibitor, as well as it might also acts as a modulator of the normal assembly of peptidoglycan, exopolysaccharides, and proteins, which leads to an inhibition of biofilm formation and the survival of the methicillinresistant strains. In fact, a molecular docking study ${ }^{48}$ demonstrated that the presence of a carboxylic acid $(\mathrm{COOH})$, but as well as the two hydroxyls $(\mathrm{OH})$ groups in the para and ortho positions of an aromatic ring (as in the case of vescalagin/castalagin) seem to play an important role in the capacity of phenolic compounds to inhibit the MRSA survival.

We then tested the cytocompatibility of the cork-derived extracts/polyphenols toward the L929 cell line by the direct contact method for $24 \mathrm{~h}$. We used concentrations down to the MIC values (Figure 7 and Figure S15 and S16) envisioning the exploitation of their antibacterial activity. Vescalagin is cytocompatible at a concentration of $0.125 \mathrm{mg} / \mathrm{mL}$, which is its MIC value (Table 1) for the MRSA and MRSE strains without eliciting cytotoxicity to eukaryotic cells. In the case of castalagin, it was also found to be cytocompatible at a
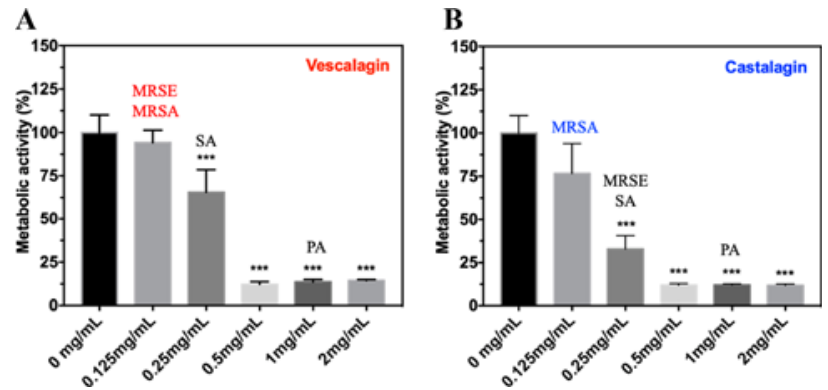

Figure 7. Cytocompatibility of (A) vescalagin and (B) castalagin toward L929 cells, evaluated by their metabolic activity using AlamarBlue. MICs are shown for each strain of bacteria, i.e., methicillin-resistant Staphylococcus epidermidis (MRSE, $0.125 \mathrm{mg} /$ $\mathrm{mL}$ ), Staphylococcus aureus (SA, $0.250 \mathrm{mg} / \mathrm{mL}$ ), methicillin-resistant Staphylococcus aureus (MRSA, $0.125 \mathrm{mg} / \mathrm{mL}$ ), and Pseudomonas aeruginosa (PA, $1.00 \mathrm{mg} / \mathrm{mL})$. * $p<0.05$, ** $p<0.01$, *** $p<0.001$ (vs $0 \mathrm{mg} / \mathrm{mL}$ of vescalagin/castalagin); $n=3$.

concentration of $0.125 \mathrm{mg} / \mathrm{mL}$, which is the MIC value only for MRSA.

After showing that vescalagin/castalagin are able to inhibit the proliferation of bacteria, especially the methicillin-resistant strains, we evaluated if they were also able to elicit the same activity once loaded into hydrogels typically used in the development of biomaterials, e.g., alginate (Figure 8). In this
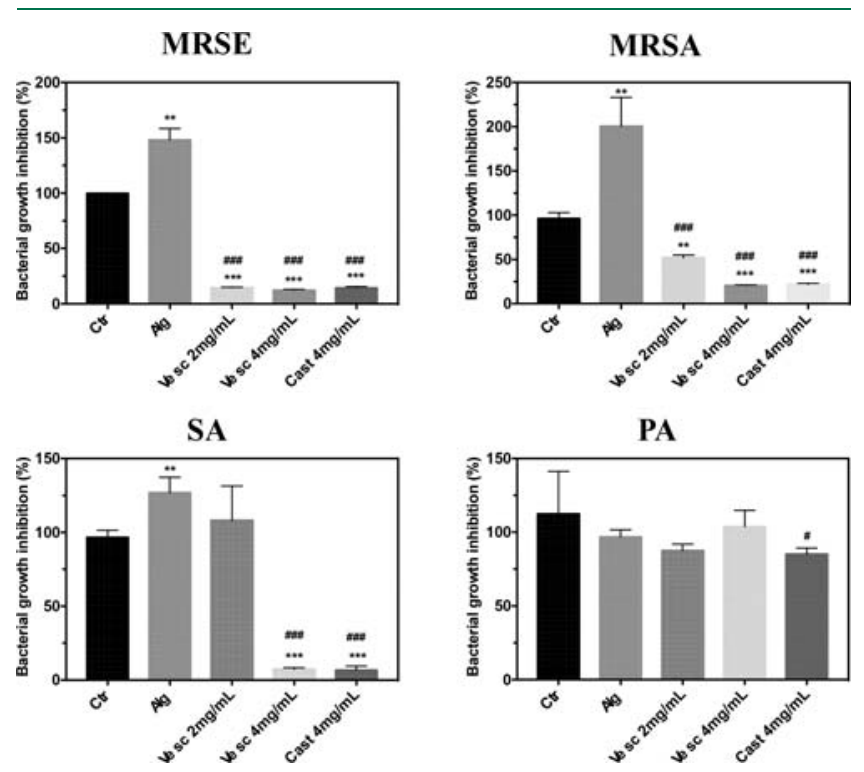

Figure 8. Ability of vescalagin/castalagin-loaded 3D hydrogels to reduce bacterial growth. Hydrogels presenting vescalagin and castalagin (loaded at a concentration of $4 \mathrm{mg} / \mathrm{mL}$ ) reduce the bacterial growth for methicillin-resistant Staphylococcus epidermidis (MRSE), Staphylococcus aureus (SA), methicillin-resistant Staphylococcus aureus (MRSA), but not for Pseudomonas aeruginosa (PA). Error bars $=\mathrm{SD}, * p<0.05$ and $* * * p<0.001$ vs $\mathrm{Alg}$ (i.e., $0 \mathrm{mg} / \mathrm{mL}$ ); $n=3$.

case, we needed to increase the vescalagin/castalagin concentrations as their spread throughout the $3 \mathrm{D}$ structure of the hydrogel drastically reduces the concentration of the compounds that are released from the hydrogel and affects the bacteria. The methicillin-resistant bacteria, i.e., MRSA and MRSE, presented susceptibility to vescalagin/castalagin already at a concentration of $2 \mathrm{mg} / \mathrm{mL}$. In the case of $S A$, we had to 
increase the concentration to $4 \mathrm{mg} / \mathrm{mL}$ to observe the same outcome. For PA, its susceptibility at both concentrations was weaker, and vescalagin/castalagin were not able to inhibit bacterial growth in the alginate hydrogels. In general, we observed the same tendency as the one observed for the direct inhibition assays, however, at higher concentrations. Despite this increment in the vescalagin/castalagin concentrations (to $2-4 \mathrm{mg} / \mathrm{mL}$ ), the loaded hydrogels were not cytotoxic toward L929 cells (Figure 9).

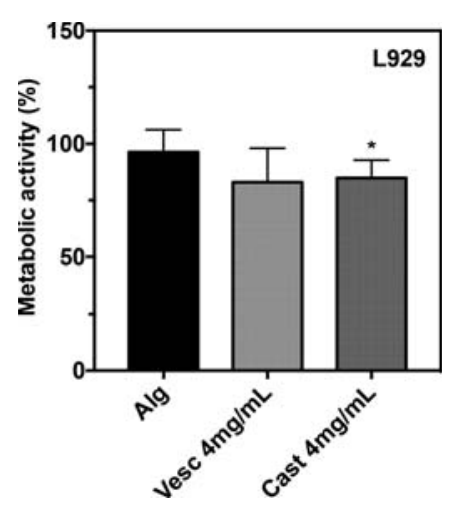

Figure 9. Cytocompatibility of 3D hydrogels loaded with vescalagin/ castalagin (at a concentration of $4 \mathrm{mg} / \mathrm{mL}$ ) toward L929 cells (seeded on the hydrogels and incubated during $24 \mathrm{~h}$ ). Metabolic activity measured using AlamarBlue assay. $* p<0.05$ vs Alg; $n=2$.

Overall, we show that vescalagin and castalagin are able to inhibit bacterial growth for methicillin-resistant Staphylococcus strains. As a proof of concept, we also demonstrated that these molecules can be loaded into 3D hydrogels as components that can be considered in the design of antibacterial biomaterials that are not cytotoxic to eukaryotic cells.

\section{CONCLUSIONS}

We investigated the potential of cork-derived extracts/ polyphenols, i.e., CWE, CWE-E, vescalagin, and castalagin, as antibacterial agents against three Gram-positive bacterial strains, i.e., MRSE, SA, and MRSA, as well as one Gramnegative bacterial strain, i.e., PA. Both vescalagin and castalagin exhibited antioxidant activity, which at the same time might be related with their capability to act against bacteria machinery in a process that is still not completely understood.

Vescalagin/castalagin presented antibacterial activity and were shown to be more effective against methicillin-resistant bacterial strains. Despite the need for more in-depth studies regarding their mode of action, vescalagin/castalagin seems to present a significant impact in the PBP2a-mediated stabilization of the peptidoglycan layer of methicillin-resistant bacteria. They seem to also be able to reduce the formation of $\beta$-sheet supramolecular arrangements necessary to maintain the bacterial cell wall integrity and biofilm formation. Moreover, based on results obtained with other polyphenols, ${ }^{46,49}$ the antibacterial activity of vescalagin might be enhanced by the combination of polyphenol and $\beta$-lactam antibiotics against MRSA, an interesting strategy that requires further testing and validation.

In general, our results can pave the way to the development of antibacterial vescalagin/castalagin-based methodologies and their future biomedical application, including the development of $3 \mathrm{D}$ antibacterial hydrogels.

\section{EXPERIMENTAL SECTION}

Extraction/Enrichment of CWE from Cork Powder, and Isolation of Vescalagin/Castalagin. The extraction of CWE followed by the purification of vescalagin and castalagin was optimized and performed following a procedure previously published. ${ }^{21}$ In short, CWE was obtained from cork powder (Amorim Cork Composites, Portugal) by Soxhlet extraction with water (under reflux) for $6 \mathrm{~h}$. After cooling, the liquid fraction was filtered and the solvent was partially removed by vacuum evaporation. The final solid extracts were recovered by freeze-drying (Figure S1, blue line). CWE$E$ was obtained from CWE by an additional step using ethanol: $5 \mathrm{mg} /$ $\mathrm{mL}$ of CWE (in water) was added to $20 \mathrm{~mL}$ of ethanol. The supernatant was collected and the solvent was partially removed by vacuum evaporation. Afterward, the final solid (CWE-E, Figure S1, red-dotted line) was recovered by freeze-drying. For the purification of vescalagin and castalagin, CWE was loaded into a semipreparative chromatographic column (Waters Atlantis OBD Prep T3, $5 \mu \mathrm{m} 19 \times$ $250 \mathrm{~mm})$, using the mobile phases $A$, water:acetic acid 98:2 (v/v), and $B$, water:acetonitrile:acetic acid 78:20:2 (v/v/v), under the following gradient: $100 \% \mathrm{~A}(t=0 \mathrm{~min})-100 \% \mathrm{~A}(t=15 \mathrm{~min})-$ $70 \% \mathrm{~A}: 30 \% \mathrm{~B}(t=30 \mathrm{~min})-100 \% \mathrm{~B}(t=35 \mathrm{~min})-100 \% \mathrm{~B}(t=50$ $\min )-100 \% \mathrm{~A}(t=52 \mathrm{~min})-100 \% \mathrm{~A}(t=57 \mathrm{~min})$. Vescalagin and castalagin were collected at their respective retention times (Figures S1 and S3). The flow rate was maintained at $5 \mathrm{~mL} / \mathrm{min}$ and the injection volume was $5 \mathrm{~mL}$. The purity of both compounds was determined by HPLC (KANUER, Germany) using a $4.6 \times 250 \mathrm{~mm}$ reverse-phase C18 column (Atlantis, Waters, UK) at flow of $1 \mathrm{~mL} /$ min, using the same mobile phases and gradients as for the semipreparative HPLC (Figure S3). Mass spectra were acquired on an electrospray ionization (ESI) mass spectrometer (MS, Water Micromass Quattro, Waters, USA) under positive-ion mode (Figures S4 and S5).

Antioxidant Activity. The radical scavenging activity was measured using 2,2-diphenyl-1-picrylhydrazyl (DPPH), following a procedure adapted from Santos et al. ${ }^{50}$ In brief, $20 \mu \mathrm{L}$ of each extract/ polyphenol was pipetted to a vial with $900 \mu \mathrm{L}$ of a DPPH solution in methanol (the initial absorbance was $0.850 \pm 0.01$ ). After vigorous vortexing, the solutions were kept in the dark for $30 \mathrm{~min}$, at $37^{\circ} \mathrm{C}$. Using a 96-well plate, we measured the absorbance $(517 \mathrm{~nm})$ in a microplate reader (Synergy HT, Bio Tek, USA). The values obtained were compared with the initial absorbance of the DPPH solution under methanol (blank). We defined five concentrations (dilutions) to produce an absorbance decrease of about 50\% (EC50). The EC50 was calculated from plotting the scavenging activity against the extract/polyphenol concentration and represents the concentration of extract/polyphenol necessary to reduce the initial DPPH concentration by $50 \%$.

Bacterial Strains and Growth Conditions. Staphylococcus aureus (SA, ATCC 25923), methicillin-resistant Staphylococcus aureus (MRSA, ATCC 700698), methicillin-resistant Staphylococcus epidermidis (MRSE, ATCC 35984) and Pseudomonas aeruginosa (PA, ATCC 27853) were obtained from the American Type Culture Collection and subcultures were incubated at $37{ }^{\circ} \mathrm{C}$ for $18-24 \mathrm{~h}$ on Tryptic soy agar (TSA, ref 610053 and 611001, Liofilchem). Suspension cultures were prepared by inoculation of single colonies in $10 \mathrm{~mL}$ of Tryptic soy broth (TSB). Afterward, for the preparation of antibacterial assays, bacteria cells were resuspended in culture media and the absorbance was adjusted to a turbidity of 0.5 McFarland scale (measured at $640 \mathrm{~nm}$ ) to an equivalent concentration of $1-2 \times 10^{8}$ colony forming units $(\mathrm{CFUs}) / \mathrm{mL}$.

Disk Diffusion Assay. The antibacterial activity was first evaluated using the disc diffusion method. The suspension cultures were prepared on TSB for $24 \mathrm{~h}$ at $37^{\circ} \mathrm{C}$ under shaking conditions at $120 \mathrm{rpm}$. Afterwards, the equivalent concentration was adjusted to $1-$ $2 \times 10^{8}$ colonies forming units (CFUs) $/ \mathrm{mL}$, followed by a homogeneous spreading onto a Muller-Hinton agar plate, (MHA, 70192, Sigma-Aldrich and 611001, Liofilchem), with a sterile swab. Sterile water was used as negative control, and gentamicin sulfate (G1914, Sigma-Aldrich) at $50 \mathrm{mg} / \mathrm{mL}$ were used as positive control. 
The disks (9999, Liofilchem) were impregnated with $10 \mu \mathrm{L}$ of each cork extracts/polyphenol, and together with the controls were placed on the inoculated plates and incubated at $37{ }^{\circ} \mathrm{C}$, during $18-24 \mathrm{~h}$. After the incubation period, the diameter of the clear zone (inhibition halo diameter) was determined to assess the antibacterial activity of each extract/polyphenol.

Minimal Inhibitory Concentrations (MICs) and Minimal Bactericidal Concentration (MBCs). The MIC and the MBC values were quantified by the microbroth dilution method. The cork extracts/polyphenols were serially diluted and dispensed into the wells $(50 \mu \mathrm{L} /$ well) which had been inoculated with $50 \mu \mathrm{L}$ of the bacterial suspension at $1-2 \times 10^{6} \mathrm{CFUs} / \mathrm{mL}$ in Muller-Hinton Broth (MHB, 70192, Sigma-Aldrich). The well plates were incubated at 37 ${ }^{\circ} \mathrm{C}$ for $24 \mathrm{~h}$ under shaking conditions. The MICs of each compound were considered to be the concentrations at which no turbidity was observed. Nevertheless, to confirm these results, we dispensed subcultures of each concentration $(10 \mu \mathrm{L})$ onto TSA for $24 \mathrm{~h}$. This assay allowed the confirmation of the MICs values and also to determine the MBCs of each extract/polyphenol. The experiments were performed in three independent experiments, each on in triplicate.

Congo Red Agar Assay. Vescalagin and castalagin were dispensed into the wells $(50 \mu \mathrm{L} /$ well) of a 96-well plate which were inoculated with $50 \mu \mathrm{L}$ of the bacterial suspension at $1-2 \times 10^{6}$ CFUs $/ \mathrm{mL}$ in MHB. The well plates were incubated at $37^{\circ} \mathrm{C}$ for $24 \mathrm{~h}$ under constant agitation. Afterward, each suspension was dispensed $(10 \mu \mathrm{L})$ onto TSA plates with $0.08 \%(\mathrm{w} / \mathrm{v})$ Congo red (C6767, Sigma-Aldrich) and supplemented with $5 \%(\mathrm{w} / \mathrm{v})$ sucrose (S0389, Sigma-Aldrich). The plates were incubated at $37{ }^{\circ} \mathrm{C}$ for $24 \mathrm{~h}$. The colony morphology and color were further analyzed, the staphylococci biofilm producer strains formed black colonies, whereas the nonbiofilm producer strains formed red colonies.

Live/Dead Assay and SEM Analysis. Each strain was evaluated after being in contact with cork extracts/polyphenols for: (i) viability through Live/Dead assay; and (ii) morphological assessment by SEM imaging. Briefly, coverslips were placed into the wells with a suspension of $50 \mu \mathrm{L}$ of the bacterial suspension at $1-2 \times 10^{6}$ CFUs/mL and $50 \mu \mathrm{L}$ of extract/polyphenol at their respective MIC concentrations. After $24 \mathrm{~h}$ of incubation, the culture medium was removed and the samples were fixed with $2.5 \%(\mathrm{v} / \mathrm{v})$ glutaraldehyde solution in PBS for $1 \mathrm{~h}$, at $4{ }^{\circ} \mathrm{C}$ in the dark. Afterward, the glutaraldehyde solution was removed, and samples were washed in PBS solution before dehydration in series of ethanol concentrations (from 10 to $100 \%$ of ethanol). After drying, the samples were platinum sputtered and visualized by SEM (FIB-SEM, Auriga Compact, Zeiss, Germany). The other group of samples were incubated using the Live/Dead BacLight Viability Kit (L23101, Life Technologies) following the protocol according to the manufacturer's instructions. Samples were analyzed by an Inverted Fluorescence Microscope (Axio Observer, Zeiss, Germany).

Inhibition of Biofilm Formation. Inocule aliquots $(300 \mu \mathrm{L})$ of each strain diluted $(1: 100)$ in TSB, were dispensed a sterile 96-well plate, during $24 \mathrm{~h}$ for the formation of the slime. The same plates were again incubated in humidified conditions at $37^{\circ} \mathrm{C}$ for $24 \mathrm{~h}$ with shaking at $150 \mathrm{rpm}$, in the presence and absence of different concentrations of vescalagin/castalagin. Following incubation, the liquid was softly removed and each well was washed three times with sterile phosphate-buffered saline (PBS; $\mathrm{pH}$ 7.3) and air-dried. Adherent bacteria were fixed with $95 \%$ ethanol and then stained with safranine (ScienCell Research Laboratories). The OD at $570 \mathrm{~nm}$ was measured with a Synergy HT microplate reader (Bio-Tek Instruments). ${ }^{47}$

Bacterial Growth Inhibition. Disk-forming solutions of alginate (Pronova UP VLVG, UniversalChemy) were prepared by dissolving alginate $2 \%(\mathrm{w} / \mathrm{v})$ in $100 \mathrm{mM} \mathrm{CaCl}_{2}$ (Laborspirit), a alginate crosslinker solution, containing different concentrations of vescalagin/ castalagin. Each bacteria strain was then added to the 3D hydrogels disks and the plates were incubated at $37{ }^{\circ} \mathrm{C}$ for $24 \mathrm{~h}$. Afterward, 100 $\mu \mathrm{L}$ of each sample was transferred to a 96 well plate and measured at a wavelength of $600 \mathrm{~nm}$ using a Synergy HT microplate reader (BioTek Instruments).

Cell Line Culture. L929 cells (passages 15-18) were maintained in DMEM, supplemented with $10 \%$ FBS (Alfagene) and $1 \%$ penicillin/streptomycin (Alfagene). Cells were plated at a concentration of 1000 cells $/ \mathrm{mL}$, in 96 -well plates, incubated at $37{ }^{\circ} \mathrm{C}$ under $5 \% \mathrm{CO}_{2}$ with the extracts/polyphenols from 2 to $0.125 \mathrm{mg} / \mathrm{mL}$, for $24 \mathrm{~h}$. For the 3D vescalagin/castalagin-loaded hydrogels, cells were seeded on the gels at a concentration of 50000 cells/gel using nonadherent 24-well plate, and incubated at $37{ }^{\circ} \mathrm{C}$ under $5 \% \mathrm{CO}_{2}$, for $24 \mathrm{~h}$. Cells were then evaluated for their cytocompatibility.

Cytocompatibility. The cytocompatibility of the cork-derived extracts/polyphenols and the 3D hydrogels loaded with vescalagin/ castalagin was evaluated after $24 \mathrm{~h}$ of culture using AlamarBlue (indicator dye BUF012B, Bio-Rad) according to the manufacturer's instructions. The fluorescence intensity of each experimental condition was measured using an excitation wavelength of $530 \mathrm{~nm}$ and an emission wavelength of $590 \mathrm{~nm}$ with a Synergy HT microplate reader (Bio-Tek Instruments). $p$-values were calculated using a onetailed $t$ test. Results are presented as mean \pm SEM of six independent experiments for each experimental condition.

\section{ASSOCIATED CONTENT}

\section{Supporting Information}

The Supporting Information is available free of charge at https://pubs.acs.org/doi/10.1021/acsbiomaterials.0c01698.

Description of the materials and methods and results related to HPLC characterization; disc diffusion assay; minimum bactericidal concentration; Live/Dead assay; Congo red assay; SEM images; and cytocompatibility assays (PDF)

\section{AUTHOR INFORMATION}

\section{Corresponding Authors}

Ricardo A. Pires - 3B's Research Group, I3Bs-Research Institute on Biomaterials, Biodegradables and Biomimetics, University of Minho, Headquarters of the European Institute of Excellence on Tissue Engineering and Regenerative Medicine, 4805-017 Barco, Guimarães, Portugal; ICVS/ 3B's-PT Government Associate Laboratory, Braga/ Guimarães, 4805-017 Barco, Guimarães, Portugal; ○ orcid.org/0000-0002-9197-0138; Email: rpires@ i3bs.uminho.pt

Ana R. Araújo - 3B's Research Group, I3Bs-Research Institute on Biomaterials, Biodegradables and Biomimetics, University of Minho, Headquarters of the European Institute of Excellence on Tissue Engineering and Regenerative Medicine, 4805-017 Barco, Guimarães, Portugal; ICVS/ 3B's-PT Government Associate Laboratory, Braga/ Guimarães, 4805-017 Barco, Guimarães, Portugal; Email: anarita.araujo@i3bs.uminho.pt

\section{Authors}

Ana C. Araújo - 3B's Research Group, I3Bs-Research Institute on Biomaterials, Biodegradables and Biomimetics, University of Minho, Headquarters of the European Institute of Excellence on Tissue Engineering and Regenerative Medicine, 4805-017 Barco, Guimarães, Portugal; ICVS/ $3 B^{\prime}$ s-PT Government Associate Laboratory, Braga/ Guimarães, 4805-017 Barco, Guimarães, Portugal

Rui L. Reis - 3B's Research Group, I3Bs-Research Institute on Biomaterials, Biodegradables and Biomimetics, University of Minho, Headquarters of the European Institute of Excellence on Tissue Engineering and Regenerative Medicine, 4805-017 Barco, Guimarães, Portugal; ICVS/3B's-PT Government 
Associate Laboratory, Braga/Guimarães, 4805-017 Barco, Guimarães, Portugal

Complete contact information is available at:

https://pubs.acs.org/10.1021/acsbiomaterials.0c01698

\section{Author Contributions}

The manuscript was written through the contributions of all authors.

\section{Notes}

The authors declare no competing financial interest.

\section{ACKNOWLEDGMENTS}

We acknowledge the financial support from the EC (FORECAST 668983 and MEPHOS 872648). A.R.A. acknowledges the "Programa Operacional Regional do Norte", "Fundo Social Europeu", and Norte2020 TERM\&SC, for her PhD grant (NORTE-08-5369-FSE-000044).

\section{REFERENCES}

(1) Nicoloff, H.; Hjort, K.; Levin, B. R.; Andersson, D. I. The high prevalence of antibiotic heteroresistance in pathogenic bacteria is mainly caused by gene amplification. Nature Microbiology 2019, 4 (3), 504-514.

(2) Abraham, W.-R. Controlling Biofilms of Gram-Positive Pathogenic Bacteria. Curr. Med. Chem. 2006, 13 (13), 1509-1524.

(3) Flemming, H.-C.; Wingender, J. The biofilm matrix. Nat. Rev. Microbiol. 2010, 8 (9), 623-633.

(4) Abee, T.; Kovács, Á. T.; Kuipers, O. P.; Van der Veen, S. Biofilm formation and dispersal in Gram-positive bacteria. Curr. Opin. Biotechnol. 2011, 22 (2), 172-179.

(5) Reichhardt, C.; Jacobson, A. N.; Maher, M. C.; Uang, J.; McCrate, O. A.; Eckart, M.; Cegelski, L. Congo Red Interactions with Curli-Producing E. coli and Native Curli Amyloid Fibers. PLoS One 2015, 10 (10), e0140388.

(6) Schembri, M. A.; Givskov, M.; Klemm, P. An Attractive Surface: Gram-Negative Bacterial Biofilms. Sci. Signaling 2002, 2002 (132), re6-re6.

(7) Coetser, S.; Cloete, T. E. Biofouling and biocorrosion in industrial water systems. Crit. Rev. Microbiol. 2005, 31 (4), 213-232.

(8) Hernández-Jiménez, E.; del Campo, R.; Toledano, V.; VallejoCremades, M. T.; Muñoz, A.; Largo, C.; Arnalich, F.; García-Rio, F.; Cubillos-Zapata, C.; López-Collazo, E. Biofilm vs. planktonic bacterial mode of growth: Which do human macrophages prefer? Biochem. Biophys. Res. Commun. 2013, 441 (4), 947-952.

(9) Lewis, K. Riddle of biofilm resistance. Antimicrob. Agents Chemother. 2001, 45 (4), 999-1007.

(10) Silhavy, T. J.; Kahne, D.; Walker, S. The bacterial cell envelope. Cold Spring Harbor Perspect. Biol. 2010, 2 (5), a000414-a000414.

(11) Gregersen, T. Rapid method for distinction of Gram-negative from Gram-positive bacteria. Eur. J. Appl. Microbiol. 1978, 5 (2), 123127.

(12) Fischetti, V. A. Surface proteins on Gram-positive bacteria. Gram-positive pathogens 2019, 19-31.

(13) Bouarab-Chibane, L.; Forquet, V.; Lantéri, P.; Clément, Y.; Léonard-Akkari, L.; Oulahal, N.; Degraeve, P.; Bordes, C. Antibacterial Properties of Polyphenols: Characterization and QSAR (Quantitative Structure-Activity Relationship) Models. Front. Microbiol. 2019, 10, No. 829.

(14) Álvarez-Martínez, F. J.; Barrajón-Catalán, E.; Encinar, J. A.; Rodríguez-Díaz, J. C.; Micol, V. Antimicrobial capacity of plant polyphenols against gram-positive bacteria: A comprehensive review. Curr. Med. Chem. 2020, 27, 2576.

(15) Tanaka, T.; Matsuo, Y.; Kouno, I. Chemistry of Secondary Polyphenols Produced during Processing of Tea and Selected Foods. Int. J. Mol. Sci. 2010, 11 (1), 14-40.
(16) Papuc, C.; Goran, G. V.; Predescu, C. N.; Nicorescu, V.; Stefan, G. Plant Polyphenols as Antioxidant and Antibacterial Agents for Shelf-Life Extension of Meat and Meat Products: Classification, Structures, Sources, and Action Mechanisms. Compr. Rev. Food Sci. Food Saf. 2017, 16 (6), 1243-1268.

(17) Porat, Y.; Abramowitz, A.; Gazit, E. Inhibition of amyloid fibril formation by polyphenols: structural similarity and aromatic interactions as a common inhibition mechanism. Chem. Biol. Drug Des. 2006, 67 (1), 27-37.

(18) Velander, P.; Wu, L.; Henderson, F.; Zhang, S.; Bevan, D. R.; $\mathrm{Xu}, \mathrm{B}$. Natural product-based amyloid inhibitors. Biochem. Pharmacol. 2017, 139, 40-55.

(19) Ngoungoure, V. L.; Schluesener, J.; Moundipa, P. F.; Schluesener, H. Natural polyphenols binding to amyloid: a broad class of compounds to treat different human amyloid diseases. Mol. Nutr. Food Res. 2015, 59 (1), 8-20.

(20) Nedumpully-Govindan, P.; Kakinen, A.; Pilkington, E. H.; Davis, T. P.; Chun Ke, P.; Ding, F. Stabilizing Off-pathway Oligomers by Polyphenol Nanoassemblies for IAPP Aggregation Inhibition. Sci. Rep. 2016, 6, 19463.

(21) Aroso, I. M.; Araujo, A. R.; Fernandes, J. P.; Santos, T.; Batista, M. T.; Pires, R. A.; Mano, J. F.; Reis, R. L. Hydroalcoholic extracts from the bark of Quercus suber L. (Cork): optimization of extraction conditions, chemical composition and antioxidant potential. Wood Sci. Technol. 2017, 51 (4), 855-872.

(22) Araújo, A. R.; Pereira, D. M.; Aroso, I. M.; Santos, T.; Batista, M. T.; Cerqueira, M. T.; Marques, A. P.; Reis, R. L.; Pires, R. A. Cork extracts reduce UV-mediated DNA fragmentation and cell death. RSC Adv. 2015, 5 (116), 96151-96157.

(23) Araujo, A. R.; Camero, S.; Taboada, P.; Reis, R. L.; Pires, R. A. Vescalagin and castalagin reduce the toxicity of amyloid-beta42 oligomers through the remodelling of its secondary structure. Chem. Commun. (Cambridge, U. K.) 2020, 56 (21), 3187-3190.

(24) Hengge, R. Targeting Bacterial Biofilms by the Green Tea Polyphenol EGCG. Molecules 2019, 24 (13), 2403.

(25) Larsen, P.; Nielsen, J. L.; Dueholm, M. S.; Wetzel, R.; Otzen, D.; Nielsen, P. H. Amyloid adhesins are abundant in natural biofilms. Environ. Microbiol. 2007, 9 (12), 3077-3090.

(26) Serra, D. O.; Mika, F.; Richter, A. M.; Hengge, R. The green tea polyphenol EGCG inhibits E. coli biofilm formation by impairing amyloid curli fibre assembly and downregulating the biofilm regulator CsgD via the sigma(E) -dependent sRNA RybB. Mol. Microbiol. 2016, 101 (1), 136-51.

(27) Halliwell, B. Are polyphenols antioxidants or pro-oxidants? What do we learn from cell culture and in vivo studies? Arch. Biochem. Biophys. 2008, 476 (2), 107-112.

(28) El Gharras, H. Polyphenols: food sources, properties and applications - a review. Int. J. Food Sci. Technol. 2009, 44 (12), 25122518.

(29) Ricardo-da-Silva, J. M.; Cheynier, V.; Souquet, J. M.; Moutounet, M.; Cabanis, J. C.; Bourzeix, M. Interaction of grape seed procyanidins with various proteins in relation to wine fining. $J$. Sci. Food Agric. 1991, 57 (1), 111-125.

(30) Naqvi, S. A. R.; Nadeem, S.; Komal, S.; Naqvi, S. A. A.; Mubarik, M. S.; Qureshi, S. Y.; Ahmad, S.; Abbas, A.; Raza, S. S.; Aslam, N. Antioxidants: Natural Antibiotics. In Antioxidants; IntechOpen: Rijeka, Croatia, 2019.

(31) Mahasenan, K. V.; Molina, R.; Bouley, R.; Batuecas, M. T.; Fisher, J. F.; Hermoso, J. A.; Chang, M.; Mobashery, S. Conformational Dynamics in Penicillin-Binding Protein 2a of MethicillinResistant Staphylococcus aureus, Allosteric Communication Network and Enablement of Catalysis. J. Am. Chem. Soc. 2017, 139 (5), 21022110.

(32) Bowden, M. G.; Chen, W.; Singvall, J.; Xu, Y.; Peacock, S. J.; Valtulina, V.; Speziale, P.; Höök, M. Identification and preliminary characterization of cell-wall-anchored proteins of Staphylococcus epidermidis. Microbiology 2005, 151 (5), 1453-1464.

(33) Vivas, N.; Laguerre, M.; Pianet de Boissel, I.; Vivas de Gaulejac, N.; Nonier, M. F. Conformational interpretation of vescalagin and 
castalagin physicochemical properties. J. Agric. Food Chem. 2004, 52 (7), 2073-8.

(34) Lambert, P. Mechanisms of antibiotic resistance in Pseudomonas aeruginosa. J. R. Soc. Med. 2002, 95 (Suppl 41), 22-26.

(35) Freeman, D.; Falkiner, F.; Keane, C. New method for detecting slime production by coagulase negative staphylococci. J. Clin. Pathol. 1989, 42 (8), 872-874.

(36) Rabin, N.; Zheng, Y.; Opoku-Temeng, C.; Du, Y.; Bonsu, E.; Sintim, H. O. Agents that inhibit bacterial biofilm formation. Future Med. Chem. 2015, 7 (5), 647-671.

(37) Hammer, N. D.; Schmidt, J. C.; Chapman, M. R. The curli nucleator protein, CsgB, contains an amyloidogenic domain that directs CsgA polymerization. Proc. Natl. Acad. Sci. U. S. A. 2007, 104 (30), 12494-12499.

(38) Sgarbossa, A. Natural biomolecules and protein aggregation: emerging strategies against amyloidogenesis. Int. J. Mol. Sci. 2012, 13 (12), 17121-37.

(39) Rohde, H.; Frankenberger, S.; Zähringer, U.; Mack, D. Structure, function and contribution of polysaccharide intercellular adhesin (PIA) to Staphylococcus epidermidis biofilm formation and pathogenesis of biomaterial-associated infections. Eur. J. Cell Biol. 2010, 89 (1), 103-111.

(40) Colvin, K. M.; Irie, Y.; Tart, C. S.; Urbano, R.; Whitney, J. C.; Ryder, C.; Howell, P. L.; Wozniak, D. J.; Parsek, M. R. The Pel and Psl polysaccharides provide Pseudomonas aeruginosa structural redundancy within the biofilm matrix. Environ. Microbiol. 2012, 14 (8), 1913-1928.

(41) Kaiser, T. D. L.; Pereira, E. M.; dos Santos, K. R. N.; Maciel, E. L. N.; Schuenck, R. P.; Nunes, A. P. F. Modification of the Congo red agar method to detect biofilm production by Staphylococcus epidermidis. Diagn. Microbiol. Infect. Dis. 2013, 75 (3), 235-239.

(42) Cao, H.; Lai, Y.; Bougouffa, S.; Xu, Z.; Yan, A. Comparative genome and transcriptome analysis reveals distinctive surface characteristics and unique physiological potentials of Pseudomonas aeruginosa ATCC 27853. BMC Genomics 2017, 18 (1), 459.

(43) Hrv, R.; Devaki, R.; Kandi, V. Evaluation of Different Phenotypic Techniques for the Detection of Slime Produced by Bacteria Isolated from Clinical Specimens. Cureus 2016, 8 (2), e505e505.

(44) Plyuta, V.; Zaitseva, J.; Lobakova, E.; Zagoskina, N.; Kuznetsov, A.; Khmel, I. Effect of plant phenolic compounds on biofilm formation by Pseudomonas aeruginosa. APMIS 2013, 121 (11), 1073-1081.

(45) Mukherjee, S.; Jemielita, M.; Stergioula, V.; Tikhonov, M.; Bassler, B. L. Photosensing and quorum sensing are integrated to control Pseudomonas aeruginosa collective behaviors. PLoS Biol. 2019, 17 (12), e3000579.

(46) Stapleton, P. D.; Taylor, P. W. Methicillin resistance in Staphylococcus aureus: mechanisms and modulation. Sci. Prog. 2002, 85 (1), 57-72.

(47) Blanco, A. R.; Sudano-Roccaro, A.; Spoto, G. C.; Nostro, A.; Rusciano, D. Epigallocatechin Gallate Inhibits Biofilm Formation by Ocular Staphylococcal Isolates. Antimicrob. Agents Chemother. 2005, 49 (10), 4339-4343.

(48) Alves, M. J.; Ferreira, I. C.; Froufe, H. J.; Abreu, R.; Martins, A.; Pintado, M. Antimicrobial activity of phenolic compounds identified in wild mushrooms, SAR analysis and docking studies. J. Appl. Microbiol. 2013, 115 (2), 346-357.

(49) Qin, R.; Xiao, K.; Li, B.; Jiang, W.; Peng, W.; Zheng, J.; Zhou, $\mathrm{H}$. The combination of catechin and epicatechin gallate from Fructus crataegi potentiates $\beta$-lactam antibiotics against methicillin-resistant Staphylococcus aureus (MRSA) in vitro and in vivo. Int. J. Mol. Sci. 2013, 14 (1), 1802-1821.

(50) Santos, S. A.; Pinto, P. C.; Silvestre, A. J.; Neto, C. P. Chemical composition and antioxidant activity of phenolic extracts of cork from Quercus suber L. Ind. Crops Prod. 2010, 31 (3), 521-526. 